CERN-TH/96-11

MPI-PhT/96-4

\title{
Naturally Light Higgs Doublet in the Spinor Representation of SUSY $S O(10)$
}

\author{
Gia Dvali* \\ CERN, CH-1211 Geneva 23, Switzerland \\ Stefan Pokorski ${ }^{\dagger}$ \\ Max-Planck-Institute für Physik, Föhringer Ring 6, 80805 \\ Munich, Germany
}

\begin{abstract}
Within the supersymmetric $S O(10)$ GUT we explore the possibility of the light Higgs doublet being a member of the 16-dimensional spinorial representation. This fact is ultimately related with the assumption that the light matter (at least partially) resides in some of the tensor representations as well. Several interesting features emerge. First, provided that the same 16-plet is responsible for the breaking $S O(10) \rightarrow S U(5)$, the heaviness of the top can automatically follow from the field content at $M_{G U T}$, without need of any flavour symmetries. Secondly, the doublettriplet splitting problem receives a new natural solution. In addition, a dimension $=5$ (Higgsino mediated) proton decay can be naturally suppressed. We construct explicit $S O(10)$ models with the above properties, with most general superpotentials under the symmetries.
\end{abstract}

${ }^{*}$ E-mail: dvali@surya11.cern.ch

${ }^{\dagger}$ On leave of absence from Institute for Theoretical Physics, Warsaw University. 


\section{Introduction}

The $S O(10)$ group has many beautiful properties which make it a promising candidate for a realistic grand unified theory (GUT). As is known, the minimal version of this theory is very constrained: each family of quarks and leptons belongs to a single spinorial irreducible representation $16^{\alpha}$-plet (where $\alpha=1,2,3$ stands for the family index) and the pair of light electroweak Higgs doublets resides in a single 10-plet. This which should lead this theory to successful predictions, unfortunately, is precisely what makes it incompatible with experiment. The reason is that the standard Yukawa coupling

$$
Y_{\alpha, \beta} 16^{\alpha} 16^{\beta} 10,
$$

'knows' only about the breaking of the electroweak symmetry (through the vacuum expectation value (VEV) of the 10-plet) and also about the 'up-down' symmetry breaking through the well-known parameter $\tan \beta$ (ratio of the 'up' and 'down' Higgs doublet VEVs). For the heaviest family this offers the exciting possibility of top-bottom Yukawa unification for the large $\tan \beta$ regime[1]. However, some prediction for the first two generations (e.g. $m_{\mu}=m_{s}$ and $m_{c} / m_{t}=m_{s} / m_{b}$ ) are a disaster. To avoid the problem, the light-fermion Yukawa couplings should receive the message about the GUT symmetry breaking at the tree level. This naturally calls for the assumption of the light matter residing not purely in three 16-plets, but also (at least partially) in some vector-like representations of $S O(10)$, which can directly couple to the GUT breaking VEVs in the superpotential [2]. There is no reason to assume that this vector-like matter should include only new $16, \overline{16}$ pairs and the tensor representations such as 45 -plet can naturally be there as well. The latter possibility automatically implies the light Higgs doublet(s) residing in the spinorial Higgs representation(s). The simplest candidate for such a Higgs is $16, \overline{16}$ pair, which at the same time is the simplest candidate for the breaking of $S O(10)$ down to $S U(5)$ and is automatically present in the minimal schemes. Of course, the 16-plet Higgs in which the light doublets reside, may not be the same as induces the breaking $S O(10) \rightarrow S U(5)$. The possibility for the light Higgs doublet to reside in 16 was usually ignored in the literature (for some exceptions see, e.g. [3]), and is the subject of the present paper. We identify at least two interesting aspects of this scenario. Especially interesting we find the situation where there is a single $16, \overline{16}$ pair in the theory which at the same time does the GUT symmetry breaking and delivers a pair of the light doublets. This offers an interesting possibility to explain the heaviness of the top quark in terms of the $S O(10)$ representation content of the matter multiplets, which are light above the GUT scale.

We construct a simple complete model of this kind in which the light Higgs doublets are also partially contained in some 10-plets. The doublet-triplet splitting is generated inside the 10-plet via the usual 'missing VEV mechanism' [4 and transmitted to the 16's through the proper mixing. The top-bottom hierarchy automatically results from the hierarchy of GUT breaking scales, even for the low values of $\tan \beta$.

However, the scenario with light doublets in 16 and $\overline{16}$ also offers a new possibility to solve naturally the doublet-triplet splitting problem in terms of the symmetries and the group structure and without any light 10's in the spectrum. Here also we construct the simplest model. In this model the pair of $16, \overline{16}$ that contains the light Higgs doublets is 
not the same as the one that breaks $S O(10)$ to $S U(5)$; therefore, in this model we do not address the question of the heaviness of the top quark. Although in our simplest models the two new aspects of the light Higgs doublets in 16, $\overline{16}$ are not simultaneously realized, this can in principle be achieved at the expense of a more complicated heavy spectrum.

\section{Naturally heavy top}

Let us address the issue of the fermion masses in the context of the light Higgses $\left(H_{u}, H_{d}\right)$ living in the 16-dimensional spinor representations $\chi, \bar{\chi}$, which at the same time breaks $S O(10)$ to $S U(5)$. As pointed out above, this implies the light matter residing (at least partially) in some tensor representations.

Let us assume for a moment that this set includes a single 45-plet with the mass $\sim M_{G}$, whereas other states are much heavier $\sim M_{\text {Planck }}$ or so (in particular this can be the case if 45 transforms under some extra symmetry broken at $M_{G}$ ). Then the relevant Yukawa couplings are:

$$
g_{\alpha} \bar{\chi} 16^{\alpha} 45+M_{45} 45^{2}
$$

where $g_{\alpha}$ are the Yukawa coupling constants and without loss of generality one can always redefine $16^{3}=16^{\alpha} g_{\alpha}\left(g_{\beta}^{2}\right)^{\frac{1}{2}}$. Thus 45 -plet couples effectively to a single $16^{3}$. Now inserting the $S U(5)$-singlet VEV of $\bar{\chi}$, we find the following mass matrix of the states that transform as 10 under $S U(5)$

$$
\overline{10}_{45}\left[10_{16^{3}} g\langle\chi\rangle+10_{45} M_{45}\right]
$$

where $g=\left(g_{\beta}^{2}\right)^{1 / 2}$ and $\langle\chi\rangle \sim M_{45}$ is an $S U(5)$-singlet VEV. Thus, one superposition $10_{\text {light }}=\left[10_{16^{3}} M_{45}-10_{45}\langle\chi\rangle\right]\left(M_{45}^{2}+g^{2}\langle\chi\rangle^{2}\right)^{\frac{1}{2}}$ stays massless at this stage and gets the tree-level mass after electroweak symmetry breaking from the coupling

$$
5_{\bar{\chi}} 10_{\text {light }} 10_{\text {light }} \text {. }
$$

This induces the mass of a single up-type quark only, naturally to be identified with the top. The rest of the matter fermions are left massless at this stage. Thus, top is the naturally the heaviest state in this picture and this is achieved without any flavour symmetry, or other input difference among families, but just due to a field content at $M_{G}$ [

Now what about the masses of the lighter fermions? Although the explanation of the complete mass pattern will not be attempted here, we will briefly mention some possibilities. One way to generate the masses of the lighter families is to assume that they come from the effective operators induced after integrating out the vector-like matter at scales larger than $M_{G}$.

We thus have to assume couplings

$$
\chi 16^{\alpha} N+\bar{\chi} 16^{\alpha} R+M_{R} R^{2}+M_{N} N^{2}
$$

where $N$ and $R$ are some heavy fields in the appropriate tensor representations contained in the products $16 \times 16$ and $16 \times \overline{16}$. $M_{N}$ and $M_{R}$ have to be understood as certain superpositions of constant mass terms with the GUT-breaking Higgs VEVs. After integrating

\footnotetext{
${ }^{1} 1 \mathrm{~A}$ similar approach describing the quark mass hierarchy in terms of the heavy field content at $M_{G}$ (without appealing to flavour symmetry) was developed in the context of $S U(6)$ GUT [5]
} 
them out, we will get effective operators of the form:

$$
\frac{1}{M_{N}} \chi \chi 16^{\alpha} 16^{\beta}+\frac{1}{M_{R}} \bar{\chi} \overline{1} 16^{\alpha} 16^{\beta}
$$

which induce the masses of the light fermions. An alternative way is to assume that the small admixture $(\sim \epsilon)$ of the light doublet resides also in $10^{\prime}$-plet Higgs. Then the lighter masses can be induced from the coupling

$$
10^{\prime} 16^{\alpha} 16^{\beta}
$$

In the next section we show that the smallness of $\epsilon$ and thus the top-bottom mass hierarchy can be directly related to the hierarchy of the $S O(10)$-breaking scales and namely $\chi / A \sim$ $\tan \beta m_{b} / m_{t}$, where $\chi$ and $A$ are two VEVs invariant under $S U(5)$ and $S U(4)_{C} \otimes S U(2)_{L} \otimes$ $U(1)_{R}$ respectively.

\section{A complete model with naturally heavy top}

We begin with the discussion of the doublet-triplet splitting mechanism. As already mentioned, in our model the light Higgs doublets are partially located in the same 16 that breaks $S O(10)$ to $S U(5)$ (to get a natural solution for the heaviness of the top quark) and partially in some 10-plet (depending on the symmetries this 10-plet may or may not be coupled to light fermions). A realistic model requires the existence of a 45-plet Higgs (hereafter denoted by $A$ ) with the VEV of the form

$$
\langle A\rangle=\operatorname{diag}[0,0,0, A, A] \otimes \epsilon
$$

where $A \sim M_{G U T}$, and each element is assumed to be proportional to the $2 \times 2$ antisymmetric matrix $\epsilon$. This VEV breaks the $G_{L, R}=S U(2)_{L} \otimes S U(2)_{R} \otimes S U(4)$ subgroup of $S O(10)$ down to $S U(2)_{L} \otimes U(1)_{R} \otimes S U(4)$ and is thus oriented along the $T_{R}^{3}$ generator of $S U(2)_{R}$. In combination with the other VEVs, say the 16-plet with non-zero SU(5)-singlet $\mathrm{VEV}$, it leads to the desired breaking $S O(10) \rightarrow G_{W}=S U(3)_{c} \otimes S U(2)_{L} \otimes U(1)_{Y}$.

We also need another 45-plet Higgs (to be denoted by $B$ ) with the VEV along the $B-L$ direction

$$
\langle B\rangle=\operatorname{diag}[B, B, B, 0,0] \otimes \epsilon
$$

in order to ensure the doublet-triplet splitting inside the 10-plet via the usual 'missing VEV' mechanism [4]. From the first glance the introduction of the second 45-plet looks somewhat unmotivated, since the group theoretically single 45-plet $A$, in combination with $\chi, \bar{\chi}$, is quite enough to break the $S O(10)$ group down to $G_{W}=S U(3)_{C} \otimes S U(2)_{L} \otimes U(1)$. However, on practice it turns out that the most general renormalizable superpotential of single 45 and 16, $\overline{1} 6$ pair does not allow for such a minimum (the only sensible minimum is the $S U(5)$-symmetric one). Thus, the introduction of extra 45-plet(s) (and even 54plet) is necessary in order to complete the breaking (see section 6). In this section we construct the superpotential which is most general under symmetries and automatically

delivers a desired VEV structure. Now let us show how the above VEV structure creates 
a light doublet in the same 16-plet as does the GUT symmetry breaking. Consider the superpotential

$$
W_{D T}=\frac{g}{2} \chi \chi 10+\frac{g^{\prime}}{2} \bar{\chi} \bar{\chi} 10+f A 1010^{\prime}+q B 10^{\prime} 10^{\prime \prime}+\frac{p}{2} \nu 10^{\prime \prime 2}
$$

where $10,10^{\prime}$ and $10^{\prime \prime}$ are three 10 -plets and $\nu$ is a gauge singlet with the $\mathrm{VEV} \sim M_{G}$. As shown below the above structure can be a natural consequence of an exact symmetry of the theory and the three 10-plets is a minimal set needed for our purposes (see eq(11)). In the full theory, the above superpotential has to be supplemented with the piece that includes the interaction of $\chi, \bar{\chi}$ with other GUT Higgses and induces its VEV in the $S U(5)$ singlet direction. This will be done below, but now let us ignore it for the moment and only remember that the components of $\chi, \bar{\chi}$ that transform as 10, $\overline{10}$ under $S U(5)$ become heavy (1) because they partially reside in the Goldstone multiplets that are eaten up by the gauge superfields of broken generators $S O(10) / S U(5)$; and (2) because of the mixing with the similar components from Higgs 45-plets. Thus, we have to discuss only the masses of the states that transform as $5, \overline{5}$ of $S U(5)$. It is not difficult to see that the mass matrices of the doublet and triplet components from $10,10^{\prime}, 10^{\prime \prime}, \chi, \bar{\chi}$ have the following form:

$$
M_{\text {doublet }}=\left(\begin{array}{cccc}
0 & g \chi & 0 & 0 \\
g^{\prime} \chi & 0 & f A & 0 \\
0 & -f A & 0 & 0 \\
0 & 0 & 0 & p \nu
\end{array}\right) ; M_{\text {triplet }}=\left(\begin{array}{cccc}
0 & g \chi & 0 & 0 \\
g^{\prime} \chi & 0 & 0 & 0 \\
0 & 0 & 0 & q B \\
0 & 0 & -q B & p \nu
\end{array}\right)
$$

Thus, the triplets are all heavy, whereas the doublet mass matrixes have one zero eigenvalue each:

$$
H_{\text {light }}=\left[H_{\chi} A-H_{10^{\prime}} g^{\prime}\langle\chi\rangle\right]\left(g^{2}\langle\chi\rangle^{2}+A^{2}\right)^{1 / 2}
$$

Thus, the admixture of the light doublet(s) in $\chi, \bar{\chi}$ and $10^{\prime}$ is controlled by the hierarchy of the symmetry-breaking scales $S O(10) \rightarrow S U(4) \otimes S U(2)_{L} \otimes U(1)$ versus $S O(10) \rightarrow S U(5)$. Say, if the latter scale is somewhat smaller, the light doublet will predominantly live in $\chi, \bar{\chi}$. As was argued above, this offers an interesting possibility for relating the top-bottom mass hierarchy to the hierarchy of the symmetry breaking.

We are now in a position to write the complete superpotential of the theory. In fact what we need now is to take care of the Higgs sector that breaks GUT symmetry and satisfies the following requirements:

(a) $W_{\text {Higgs }}$ should be most general under symmetries;

(b) there should be no 'fine-tuning';

(c) it should allow for the $G_{w}$-Symmetric SUSY minimum in which the only light states are the ones of the minimal supersymmetric standard model ( + possibly some $G_{W}$-singlets) and a pair of light doublets residing at least partially in Higgs 16-plets.

The Higgs superpotential includes the chiral superfields in the following $S O(10)$ representations: $S, X, Y$-singlets; $\Sigma$-54-plet, $A, B, C$-45-plets; $\chi, \bar{\chi}-16, \overline{16}$-plets and three 10 -plets $10,10^{\prime}, 10^{\prime \prime}$. We also introduce two singlets $\nu, \nu^{\prime}$ and the one 45-plet in the matter sector. 
The Higgs superpotential has the form:

$$
W_{H i g g s}=W_{G U T}+W_{D T} .
$$

The two parts are given as

$$
\begin{aligned}
W_{G U T} & =\frac{\sigma}{4} S \operatorname{Tr} \Sigma^{2}+\frac{h}{6} \operatorname{Tr} \Sigma^{3}+\frac{1}{4} \operatorname{Tr}\left(a \Sigma+M_{a}+a^{\prime} S\right) A^{2}+\frac{1}{4} \operatorname{Tr}\left(b \Sigma+M_{b}+b^{\prime} S\right) B^{2} \\
& +\frac{1}{2} \operatorname{Tr}\left(a^{\prime \prime} X A+b^{\prime \prime} Y B\right) C+\frac{g_{c}}{2} \bar{\chi} C \chi+\left(r_{3} \nu+r_{4} \nu^{\prime}\right) \operatorname{Tr} C^{2}+M^{2} S \\
& +\frac{M^{\prime}}{2} S^{2}+\frac{\kappa}{3} S^{3}
\end{aligned}
$$

and

$$
W_{D T}=g \chi 10 \chi+\bar{g} \bar{\chi} 10 \bar{\chi}+f A 10^{\prime} 10+q B 10^{\prime} 10^{\prime \prime}+r \nu 10^{2}+\left(r_{1} \nu+r_{1} \nu^{\prime}\right) 10^{\prime \prime 2}
$$

This form is strictly natural, since it is the most general compatible with the $Z_{2}^{A} \otimes Z_{2}^{B} \otimes$ $U(1)^{C}$ global symmetry under which the chiral superfields transform as follows: under $Z_{2}^{A}$

$$
\left(A, X, 10^{\prime}, 10^{\prime \prime}\right) \rightarrow-\left(A, X, 10^{\prime}, 10^{\prime \prime}\right)
$$

under $Z_{2}^{B}$

$$
\left(B, Y, 10^{\prime \prime}\right) \rightarrow-\left(B, Y^{\prime} 10^{\prime \prime}\right)
$$

and under $U(1)^{C}$

$$
\begin{aligned}
\left(C, 10,10^{\prime \prime}\right) & \rightarrow e^{i 2 \alpha}\left(C, 10,10^{\prime \prime}\right) \\
(\chi, \bar{\chi}) & \rightarrow e^{-i \alpha}(\chi, \bar{\chi}) \\
\left(X, Y, 10^{\prime}\right) & \rightarrow e^{-i 2 \alpha}\left(X, Y^{\prime} 10^{\prime}\right) \\
16^{\beta} & \rightarrow e^{i \alpha} 16^{\beta} \\
\left(\nu, \nu^{\prime}\right) & \rightarrow e^{-i 4 \alpha}\left(\nu, \nu^{\prime}\right) .
\end{aligned}
$$

Besides we also assume 'matter parity' under which $16^{\alpha}$ and 45 change sign and all other superfields are invariant.

The above superpotential admits the following supersymmetric ( $F$-flat and $D$-flat) minimum with an unbroken $G_{W}$ symmetry:

$$
\begin{aligned}
\Sigma & =\operatorname{diag}(2,2,2,2,2,2,-3,-3,-3,-3) \Sigma \quad \text { where } \Sigma=\frac{b^{\prime} M_{a}-a^{\prime} M_{b}}{3 a b^{\prime}+2 b a^{\prime}} \\
A & =\operatorname{diag}[0,0,0, A, A] \otimes \epsilon \\
B & =\operatorname{diag}[B, B, B, 0,0] \otimes \epsilon \\
\chi & =\bar{\chi}=\chi|+,+,+,+,+\rangle \text { where } \chi^{2}=-\frac{a^{\prime \prime}}{g_{c}} X A=-\frac{b^{\prime \prime}}{g_{c}} Y B \\
S & =-\frac{2 b M_{a}+3 a M_{b}}{3 a b^{\prime}+2 b a^{\prime}} \\
10 & =10^{\prime}=10^{\prime \prime}=C=\nu=0
\end{aligned}
$$


According to the standard notations (e.g. see [6]) the $S U(5)$ singlet component of 16 is denoted by $|+,+,+,+,+\rangle$, where each ' + ' refers to an eigenvalue of the respective Cartan subalgebra generator. The two quantities $A$ and $B$ are determined from the two equations:

$$
\begin{aligned}
& 10\left(S \sigma \Sigma-h \Sigma^{2}\right)-a A^{2}+b B^{2}=0 \\
& 15 \sigma \Sigma^{2}+a^{\prime} A^{2}+\frac{3}{2} b^{\prime} B^{2}+M^{2}+M^{\prime} S+\kappa S^{2}=0
\end{aligned}
$$

Note that the absolute VEVs of the singlets $X$ and $Y$ are undetermined in the SUSY limit, and only their ratio, $\frac{X}{Y}=\frac{b^{\prime \prime} B}{a^{\prime \prime} A}$, is determined. The VEV of $\nu^{\prime}$ is also undetermined in the SUSY limit and we assume that it will be fixed $\sim M_{G}$ after supersymmetry breaking. Then, according to the analysis of section 2, the light doublet pair is partially residing in $\chi, \bar{\chi}$ and $10^{\prime}$ representations, and its admixture is controlled by the hierarchy of the scales $(\langle\chi\rangle / A$ ratio). Since the $\langle\chi\rangle$ VEV parametrizes a SUSY flat direction, in the SUSY limit the theory admits two realistic vacuum states: in the limit $\langle\chi\rangle>>A$ it recovers an 'old' top-bottom unification prediction of the minimal $S O(10)$, whereas in the other case, $\langle\chi\rangle<<A$, it leads to the heavy top for the low $\tan \beta$ regime.

\section{Higgsino-induced proton decay}

Considered scheme allows for the natural suppression of the coloured Higgsino-mediated (dimension=5) proton decay [7], which is usually a problem in the standard approaches (for some alternative solutions within the $S O(10) \mathrm{GUT}$ see, e.g.[B]). As it is known the dangerous dimension $=5$ operators can occur only if there is a supersymmetric (chirality flip) mass insertion $M_{T} \bar{T} T$ which mixes the colored triplet partners $T, \bar{T}$ of $H_{u}$ and $H_{d}$ Higgs doublets respectively. To study suppression we have to identify $T, \bar{T}$ states that are coupled to light matter fermions. This depends on the way the light family masses are generated and, therefore, we separately discuss two above-mentioned possibilities.

One possibility is that $10^{\prime}$ is decoupled from the quarks and leptons and the masses are generated from the couplings (4) and (6) (which are low energy remnant of (2) and (5) respectively). In this case the only colored triplets coupled to light matter are $\bar{T}_{\chi}$ and $T_{\bar{\chi}}$ states from $\chi$ and $\bar{\chi}$ respectively. Due to (11) these triplets have no supersymmetric crossing mass term and thus, there are no Higgsino induced dimension $=5$ operators.

Now assume that at least some of the light fermion masses are induced from the coupling (7). Then, there is another pair of coloured triplets $T_{10^{\prime}}, \bar{T}_{10^{\prime}}$ coupled to the quark and lepton superfields. However, as it can be seen from (11), their chirality flip mass term is only induced due to their mixing with $10^{\prime \prime}$ (and not directly with each other) and, therefore, for $\nu<<q B$ it is suppressed by an additional factor $\sim \frac{\nu}{q B}$. Presumably we can not take $\nu$ arbitrarily small (since it measures a mass of the doublet component from $10^{\prime \prime}$ and this in general can affect the unification of gauge couplings), but even assuming it one or two orders of magnitude below $M_{G}$ one can significantly improve the situation with respect to a standard case. 


\section{A model for the doublet-triplet splitting without a light 10- plet}

In the previous section we have constructed a simple model with emphasis on the naturally heavy top quark. This is achieved by requiring the light Higgs doublet to be in the same 16 as breaks $S O(10)$. To obtain doublet-triplet splitting in this model with only one pair $16, \overline{16}$, the simplest possibility is to introduce also a 10-plet. The doublet-triplet splitting in the 10-plet is transmitted to the 16-plet and the light Higgs doublet is partially in 16 and partially in 10 . The mixing is controlled by $A /\langle\chi\rangle$ which at the same time can give heavy top even for low $\tan \beta$. In this section we put aside the problem of the heavy family quark masses (we assume it can be solved by one of the known (or unknown!) mechanisms) and construct an explicit model where the ansatz about light Higgs doublet in $16, \overline{16}$ is used for a non-standard solution to the doublet-triplet splitting. We propose a mechanism that makes use solely of 16-plets. One may ask what are the motivations for avoiding light doublets residing in the 10-plet Higgs. First, the scenario without light 10plets is logically different possibility which is certainly worth studying. Secondly, we think that the introduction of a heavy vector-like matter (which seems in any case is necessary for the avoiding the wrong mass relations) in this scenario looks more motivated than in the standard versions (with light doublets in 10-plet): in the former case quark and lepton masses simply can not be generated without exchange of heavy vector-like states (see eq(5)), whereas in the later case they can be induced easily.

For the Higgs spectrum we take again a 45 -plet $A$ with the $T_{3 R} \mathrm{VEV}$, a pair $\chi, \bar{\chi}$ of $16, \overline{16}$ which breaks $S O(10)$, a pair $\psi, \bar{\psi}$ of $16, \overline{16}$ with light Higgs doublet and another heavy Higgs 45-plet $\Phi$.

Then, the piece of the superpotential responsible for the doublet-triplet splitting has the form:

$$
W_{D T}=g_{a} \bar{\psi} A \psi+g_{\Phi} \chi \Phi \bar{\psi}+\overline{g_{\Phi}} \bar{\chi} \Phi \psi+M \operatorname{Tr} \Phi^{2} .
$$

The doublet-triplet splitting mechanism can be easily understood in terms of the $G_{L R}$ invariant decomposition of 45 and 16 representations

$$
\begin{aligned}
16 & =(2,1,4)+(1,2, \overline{4}) \\
45 & =(1,3,1)+\ldots
\end{aligned}
$$

Now, since the VEV of $A$ transforms as $(1,3,1)$ it can give masses only to the right-hand doublet states in $\psi, \bar{\psi}$. Thus, all the left-handed states $((2,1,4)$ fragment) stay massless at these stage. This are the states with quantum numbers of Higgs doublets $H, \bar{H}$ and left-handed quark doublets $Q, \bar{Q}$. However, $Q, \bar{Q}$ states transform as $10, \overline{10}$ under $S U(5)$ and therefore they are mixed with the similar states from $\Phi$ through the $\chi, \bar{\chi}$ VEV and become heavy. In contrast, the $H, \bar{H}$ have no partners in $\Phi$ (which includes no component transforming as $5, \overline{5}$ under $S U(5))$ and thus stay massless.

We now proceed to construct an explicit model with the proper GUT potential. The Higgs superpotential includes the chiral superfields in the following $S O(10)$ representations: $S, X, Y \equiv$ singlets; $\Sigma \equiv 54$-plet; $A, B, C, \Phi \equiv 45$-plets; $\chi, \bar{\chi}, \psi, \bar{\psi} \equiv 16, \overline{16}$-plets and $F \equiv 10$-plet. The superpotential has the form:

$$
W_{\text {Higgs }}=W_{G U T}+W_{D T}
$$


where

$$
\begin{aligned}
W_{G U T} & =\frac{\sigma}{4} S \operatorname{Tr} \Sigma^{2}+\frac{h}{6} \operatorname{Tr} \Sigma^{3}+\frac{1}{4} \operatorname{Tr}\left(a \Sigma+M_{a}+a^{\prime} S\right) A^{2}+\frac{1}{4} \operatorname{Tr}\left(b \Sigma+M_{b}+b^{\prime} S\right) B^{2} \\
& +\frac{1}{2} \operatorname{Tr}\left(a^{\prime \prime} X A+b^{\prime \prime} Y B\right) C+\frac{g_{c}}{2} \bar{\chi} C \chi+M^{2} S+\frac{M^{\prime}}{2} S^{2}+\frac{\kappa}{3} S^{3}
\end{aligned}
$$

and

$$
W_{D T}=g_{f} \chi F \chi+\overline{g_{f}} \bar{\chi} F \bar{\chi}+g_{\Phi} \bar{\psi} \Phi \chi+\overline{g_{\Phi}} \bar{\chi} \Phi \psi+g_{a} \bar{\psi} A \psi+\rho X \operatorname{Tr} \Phi^{2}
$$

Again, this form is strictly natural, since it is the most general that is compatible with the $Z_{4}^{A} \otimes Z_{2}^{B} \otimes U(1)^{C}$ global symmetry under which the chiral superfields transform as follows: under $Z_{4}^{A}$

$$
\begin{aligned}
& (A, X) \rightarrow-(A, X) \\
& (\psi, \bar{\psi}) \rightarrow i(\psi, \bar{\psi}) \\
& \Phi \rightarrow-i \Phi
\end{aligned}
$$

under $Z_{2}^{B}$

$$
(B, Y) \rightarrow-(B, Y)
$$

and under $U(1)^{C}$

$$
\begin{aligned}
(C, F) & \rightarrow e^{i 2 \alpha}(C, F) \\
(\chi, \bar{\chi}) & \rightarrow e^{-i \alpha}(\chi, \bar{\chi}) \\
(X, Y) & \rightarrow e^{-i 2 \alpha}(X, Y) \\
\Phi & \rightarrow e^{i \alpha} \Phi
\end{aligned}
$$

We assume that all mass scales in $W_{G U T}$ are $\sim M_{G U T}$ and all coupling constants are of the order of 1.

The standard procedure shows that the above superpotential admits the following supersymmetric ( $F$-flat and $D$-flat) minimum with an unbroken $G_{W}$ symmetry:

$$
\begin{aligned}
\Sigma & =\operatorname{diag}(2,2,2,2,2,2,-3,-3,-3,-3) \Sigma \quad \text { where } \Sigma=\frac{b^{\prime} M_{a}-a^{\prime} M_{b}}{3 a b^{\prime}+2 b a^{\prime}} \\
A & =\operatorname{diag}[0,0,0, A, A] \otimes \epsilon \\
B & =\operatorname{diag}[B, B, B, 0,0] \otimes \epsilon \\
\chi & =\bar{\chi}=\chi|+,+,+,+,+\rangle \text { where } \chi^{2}=-\frac{a^{\prime \prime}}{g_{c}} X A=-\frac{b^{\prime \prime}}{g_{c}} Y B \\
S & =-\frac{2 b M_{a}+3 a M_{b}}{3 a b^{\prime}+2 b a^{\prime}} \\
\psi & =\bar{\psi}=F=\Phi=C=0
\end{aligned}
$$

As was shown above, in the given vacuum $W_{G U T}$ delivers a pair of the light doublets from the $\psi, \bar{\psi}$ multiplets. Once again, this is because $\psi, \bar{\psi}$ states get their masses from only the two sources: through the VEV of $A$ and via mixing with the heavy $\Phi$ through the $\chi, \bar{\chi}$ VEV. Now, the $A$ VEV leaves all $S U(2)_{L}$-doublet states in $\psi, \bar{\psi}$ massless. This 
is because all these states are zero eigenvalues of the $T_{3 R}$ generator. These are the states with quantum numbers of $Q, \bar{Q}$ and $H, \bar{H} . Q, \bar{Q}$ components of $\bar{\psi}, \psi$ are mixed with the similar components of $\Phi$ through the VEVs of $\chi, \bar{\chi}$ and become heavy. In contrast, 45-plet $\Phi$ has no colour singlet weak doublet component and thus $H, \bar{H}$ states are massless. All other $G_{W}$ non-singlet states from $W_{G U T}$ have a GUT scale mass.

\section{Why $W_{G U T}$ ?}

We have discussed in detail the structure of $W_{D T}$ and $W_{Y u k a w a}$ in the previous sections and each term there plays a well-defined role in the doublet-triplet splitting mechanism or in the fermion mass generation respectively. They have an obvious property of each term including at least two of the superfields with a vanishing VEVs in the phenomenologically acceptable SUSY vacuum. Thus, $W_{D T}$ and $W_{Y u k a w a}$ cannot affect the configuration of the VEVs in this vacuum and the symmetry breaking pattern is solely decided by $W_{G U T}$. Now what about the structure of $W_{G U T}$ ? From the first glance its form looks rather ad hoc and non-minimal. However, the more careful analysis shows that this form is one of the simplest choices that can lead to the desired breaking of $S O(10)$ to $G_{W}$ and deliver the VEV structure necessary for the doublet-triplet splitting. In this section we will justify the form of the Higgs superpotential as being one of the simplest possibilities for the natural solution of the doublet-triplet splitting problem in $S O(10)$. Below we will show why the simpler structures do not work. We will also confront the Higgs sectors needed for the natural doublet-triplet splitting via two different mechanisms, with the light doublet appearing in 10-plets (Dimopoulos-Wilczek mechanism) or 16-plet. As we know, these mechanisms require the existence of the 45-plet Higgs $A$ with the VEV along the $B-L$ or $T_{3 R}$ directions. (as discussed in section 3, a combination of the two is also possible) and the key point is the existence (among other terms) of the couplings $A_{B-L} 10^{\prime} 10$ or $A_{T_{3 R}} \overline{16}_{\text {Higgs }} 16_{\text {Higgs }}$.

Imagine therefore that the GUT sector includes the only two representations: a 45plet $A$ and a $16, \overline{16}$ pair $\chi, \bar{\chi}$. This set would be a very economical and simplest choice, since it is sufficient group theoretically for the desired symmetry breaking as well as for the desired VEV structure. Unfortunately, this simplest possibility does not work, since the $G_{W}$-symmetric state never corresponds to a SUSY minimum of the most general superpotential with an arbitrary number of gauge singlets included

$$
W=\frac{1}{4} M_{A} \operatorname{Tr} A^{2}+\frac{1}{2} g \bar{\chi} A \chi+M_{\chi} \bar{\chi} \chi+(\text { singlet self }- \text { couplings })
$$

where $M_{A}$ and $M_{\chi}$ have to be understood as the arbitrary (up to a symmetries) superpositions of the constant mass terms and the gauge-singlet superfields. The heart of the problem is in the $F$-flatness condition for the 45-plet $\left(\sigma_{k i}\right.$ are $S O(10)$ generators in the spinor representation)

$$
F_{A_{i k}}=M_{A} A_{k i}+g \bar{\chi} \sigma_{k i} \chi=0,
$$

which tells us that the only vacuum with unbroken $G_{W}$ is an $S U(5)$-symmetric one. The group theoretical reason for this is that the only acceptable maximal little group of 16 is $S U(5)$. 
The introduction of another 45-plet $B$ does not help much. To see this, first notice that a necessary requirement is that an intersection of two VEVs $A$ and $B$ should break group to $S U(3)_{c} \otimes S U(2)_{L} \otimes U(1)_{c} \otimes U(1)_{R}$, meaning that the matrix

$$
\langle A\rangle+\theta\langle B\rangle=\operatorname{diag}\left[V, V, V, V^{\prime}, V^{\prime}\right] \otimes \epsilon
$$

must have the full diagonal occupied $\left(V, V^{\prime} \neq 0\right)$ for some non-zero $\theta$. If this condition is not satisfied, the situation is effectively reduced to the case with a single 45-plet. Next, let us point out that no matter whether we want to split doublet-triplet masses inside the 10-plet or 16-plet Higgs, the $A$ and $B$ must have different transformation properties under some symmetry; otherwise the doublet and triplet mass matrices would have the same zeros and doublet-triplet splitting would be impossible without fine-tuning. Thus, only one of the 45 -plets can couple to $\chi, \bar{\chi}$ and we are lead to the generic superpotential

$W=\frac{1}{4} M_{A} \operatorname{Tr} A^{2}+\frac{1}{4} M_{B} \operatorname{Tr} B^{2}+\frac{1}{2} M_{A B} \operatorname{Tr} A B+\frac{1}{2} g \bar{\chi} A \chi+M_{\chi} \bar{\chi} \chi+($ singlet self - couplings $)$

where, as before, each mass parameter has to be understood as a whatever combination of singlets and constant mass terms allowed by symmetries that we will not specify here. This form is enough to point out the problem, since the $F$-flatness requirement among others implies the following conditions

$$
\begin{aligned}
& F_{A_{i k}}=M_{A} A_{k i}+M_{A B} B_{k i}+g \bar{\chi} \sigma_{k i} \chi=0 \\
& F_{B_{i k}}=M_{B} B_{k i}+M_{A B} A_{k i}=0
\end{aligned}
$$

Immediately we see that at best there are two possibilities (with $G_{W}$ unbroken): either $M_{B}$ and $M_{A B}$ both vanish at the minimum, and thus $A=S U(5)$-singlet and $B=$ undetermined; or

$$
B_{k i}=-\frac{M_{A B}}{M_{B}} A_{k i}=\frac{g M_{A B}}{M_{A} M_{B}-M_{A B}^{2}} \bar{\chi} \sigma_{k i} \chi=S U(5)-\text { singlet }
$$

Clearly none of the two is acceptable.

The next logical step is to introduce 54-plet $\Sigma$, which is the lowest dimensional representation that contains a singlet of $G_{W}$, but no singlet of $S U(5)$. Unfortunately, $\Sigma$ can not substitute one of the 45-plets, since it cannot couple to the 16-plets (there is no 54 contained in either of the direct products $16 \otimes 16$ or $\overline{16} \otimes 16)$. Therefore, the system with 54,45 and $16, \overline{16}$, although it can successfully break the $S O(10)$ to $G_{W}$, can never deliver a desired VEV for 45-plet. Thus, $\Sigma$ has to be introduced on top of the $A, B, \bar{\chi}, \chi$ states. For constructing the superpotential, we have to remember that: (1) as pointed above, $A$ and $B$ must transform differently under some symmetry, and (2) superpotential must contain a crossing coupling $\operatorname{Tr} \Sigma A B$ or $M_{A B} \operatorname{Tr} A B$. The second condition is needed in order to eliminate the unwanted massless pseudo-Goldstone states, which otherwise would be presented since, without a direct cross coupling, $A$ and $B$ can communicate only through the $S O(6) \otimes S O(4)$-invariant VEV of $\Sigma$ and, consequently, the correct vacuum would have an accidental global $[S O(6) \otimes S O(4)]_{A} \otimes[S O(6) \otimes S O(4)]_{B}$ degeneracy. 
Thus, the superpotential should have the following generic form

$$
\begin{aligned}
W & =M_{\Sigma} \operatorname{Tr} \Sigma^{2}+h \operatorname{Tr} \Sigma^{3}+\operatorname{Tr}\left[\left(a A^{2}+b B^{2}+c A B\right) \Sigma\right]+\frac{1}{4} M_{A} \operatorname{Tr} A^{2}+\frac{1}{4} M_{B} \operatorname{Tr} B^{2} \\
& +\frac{1}{2} M_{A B} \operatorname{Tr} A B+\frac{1}{2} g \bar{\chi} A \chi+M_{\chi} \bar{\chi} \chi+(\text { singlet self }- \text { couplings })
\end{aligned}
$$

where at least one of the two constants $a$ and $b$ should be zero. It is not difficult to find the combination of the symmetries and the singlet fields, which allow for the desired symmetry breaking and the $A$-plet having the VEVs in the $B-L$ or $T_{3 R}$ direction (or in both). However, the problem is that whenever an $S U(5)$-singlet from $\chi$ gets a VEV, the $F$-flatness condition implies

$$
M_{\chi}+3 g A=0 \quad \text { or } \quad M_{\chi}+2 g A=0
$$

for the $A \sim B-L$ and $A \sim T_{3} R$ cases, respectively. First of all, this immediately means that we cannot extract the light doublet from the same $\chi, \bar{\chi}$, because in these two cases they get the mass equal to $2 M_{\chi}$ and $M_{\chi}$, respectively. However, this is not a major problem, just the fact that the quantity $M_{\chi}$ at the minimum must be non-zero. This quantity must transform identically to $A$ under any symmetry other than $S O(10)$ and thus, it is very difficult to prevent a multiplet in which doublet-triplet splitting is induced by the VEV of $A$ from having a large $S O(10)$ invariant mass. One way to improve the situation could be to assume that $M_{\chi}$ is a superposition of several singlets which, being coupled to $10^{\prime} 10$ or $\overline{16}_{\text {Higgs }} 16_{\text {Higgs }}$, exactly compensate each other. Such a solution is possible, but we think that a much simpler way is to introduce another 45-plet $C$ with vanishing $\mathrm{VEV}$ in the right vacuum. Thus, the system with $\Sigma, A, B, C, \chi, \bar{\chi}$ corresponds to the simplest possibility and we are lead to the $W_{G U T}$ discussed in the previous sections.

\section{Summary}

Model building at the GUT scale is useful if taken with the proper attitude: as an illustration of certain general possibilities to solve the existing physical (or technical) problems in the framework of GUTs. On the other hand, it is not sensible to aim already now at the 'true' theory. In this paper we have shown that light Higgs doublets in 16, $\overline{16}$ of $S O(10)$ is a viable alternative, which may explain the heaviness of the top quark and offer a new mechanism for doublet-triplet splitting. We have also shown that it is easy to construct self-consistent (and relatively simple) models with these properties.

\section{Acknowledgements}

S.P. was partially supported by the Polish committee for Scientific Research and by the EU grant Flavourdynamics.

\section{References}

[1] M.Olechowski and S.Pokorski, Phys.Lett. B214 (1988) 393; B.Ananthanarayan, G.Lazarides, and Q.Shafi, Phys.Rev. D44 (1991) 1613. 
[2] e.g. see, G.Anderson, S.Dimopoulos, L.J.Hall, S.Raby, and G.Starkman, Phys. Rev. D49 (1994) 3660. For the early work in this direction, see C.D.Froggatt and H.B.Nielsen, Nucl.Phys.B147 (1979) 277; Z.G.Berezhiani, Phys.Lett. B129 (1983) 99; S.Dimopoulos, ibid.B129 (1983) 417.

[3] S.M.Barr, Phys.Rev. D24 (1981) 1895; Z.G.Berezhiani, Sov. J. Nucl. Phys. 42(5)(1985) 825.

[4] S.Dimopoulos and F.Wilczek, in Proceedings of the Erice Summer School, ed. A.Zichichi (Publisher, City 1981). For a recent discussion, see K.S.Babu and S.M.Barr, Phys.Rev. D50 (1994) 3529; L.J.Hall and S.Raby, Phys.Rev. D51 (1995) 6524; D.G.Lee and R.N.Mohapatra, Phys.Rev. D51 (1995) 1353; K.S.Babu and R.N.Mohapatra, Phys.Rev.Lett. 74 (1995) 2418.

[5] R.Barbieri, G.Dvali, A.Strumia, Z.Berezhiani and L.Hall, Nucl. Phys. B432 (1994) 49.

[6] F.Wilczek and A.Zee, Phys.Rev. D25 (1982) 553.

[7] S.Weinberg, Phys.Rev D26 (1982) 287; N.Sakai and T.Yanagida, Nucl.Phys. B197 (1982) 533.

[8] G.Dvali, Phys.Lett. B287 (1992) 101; Preprint CERN-TH/95-288, in Phys.Lett. (to appear); K.S.Babu and S.M.Barr, Phys.Rev. D48 (1993) 5354. 